\title{
Inversión uterina aguda
}

\section{Reporte de un caso}

\author{
Por el doctor J. Agustín Pachón Ortíz
}

En el diagnóstico diferencial del shock obstétrico, así como en el de las hemorragias del alumbramiento y del post-partum, es necesario tener en cuenta la inversión uterina aguda, que. siendo una entidad rara, es una causa importante tanto de hemorragia como de shock.

De todos es sabido, y quizá por pocos observado, el fenómeno de la inversión uterina que consiste en la vuelta de dentro hacia afuera del cuerpo uterino, a la manera de un dedo de guante, invaginándose sobre sí misma.

Clasificación.- Según la época en que se reconozca se divide en aguda o crónica. El concepto de "agudeza" es variable según los autores: para Curtis (1) las de menos de un mes son agudas, las de más de un mes, crónicas. Para Titus (2), la aguda es la que se observa inmediatamente en relación con el parto, y la crónica es en la que persiste el útéro invertido algún tiempo después del parto, habiéndose recobrado la paciente de los síntomas agudos. Para Kellog (citado por Titus), se llama aguda la que se descubre antes de que haya habido contracción del anillo cervical (lo que se produce alrededor de 30 minutos después de efectuada la inversión). Subaguda, aquella que se reconoce prontamente después de que se ha formado el anillo de contracción cervical. Crónica, comienza después de un lapso de 3 a 4 semanas de ocurrida la inversión aguda que no fue reconocida.

Personalmente nos parece mejor esta última, pues sobre ella se basan en parte las modalidades en el plan terapéutico que más adelante veremos.

En lo que se refiere al grado de la inversión se la divide en completa e incompleta. Para unos se llama completa cuando el fondo ha pasado a través del cuello; si no, es incompleta. Para 
otros es incompleta cuando el útero invertido queda en la vagina y completa cuando se insinúa en el orificio vaginal o aun sale fuera de la vulva. M. L. Pérez (3), la clasifica en: local, si se limita a pequeñas porciones de la pared; parcial, si el fondo invertido no pasa del anillo de Bandl; incompleta, cuando el fondo alcanza el orificio externo del cuello; completa, cuando llega a la vagina ocupándola, y total, si hay salida del útero invertido al exterior llevándose consigo las paredes vaginales.

Desde un punto de vista práctico de diagnóstico nos parece que ésta última es demasiado detallada y que si pasan desapercibidas las incompletas con mayor razón las locales, las parciales, etc., además estas denominaciones parecen ser distintos estados de evolución del mecanismo productor.

Así que la más sencilla nos parece que es la división en incompleta y completa, esta última con o sin prolapso, según que arrastre consigo las paredes vaginales o no.

Frecuencia.- Según datos estadísticos citados por Curtis (1) del New York Lyng-in Hospital, Boston Lyng-in Hospital y California University, la frecuencia en dichos hospitales fue de 1 por 4 mil a 1 por 8 mil partos, en promedio, 1 por 6 mil.

Harer y Sharkey presentaron 15 casos ocurridos en Filadelfia en un período de ocho años, dando una proporción de 1 pör 16.240 partos.

Porbodh Das (citado por De Lee (4) en una recopilación de 297 casos de hospitales de Estados Unidos, Inglaterra y la India da los siguientes datos:

En hospitales americanos: $1 \times 23.127$ partos.

En hospitales británicos: 1 × 27.992 partos.

En hospitales de la India: 1 x 8.537 partos.

Como se ve, la frecuencia es baja. A esto contribuyen tres factores: en parte, su rareza; en parte, la falta de reconocimiento, y finalmente, renuencia de los médicos a publicar tan desgraciada ocurrencia.

Etiología.- Se acepta, aun cuando el acuerdo no es unánime, que existe la inversión espontánea, 38 a $40 \%$ de los casos; $50 \%$ en una serie de 14 casos presentados por G. J. Quigley (5), y la causada por falta de técnica obstétrica, que podriamos denominar "provocada" y que para algunos como Titus (2) sería el casi 100 por 100 de los casos. 
G. J. Quigley (5) relata un caso de inversión "intencional", llevado a cabo bajo anestesia general, con el fin de remover una placenta ácreta parcial, logrado lo cual redujo la inversión con éxito completo.

Se pueden considerar causas predisponentes y causas determinantes tanto de las espontáneas como de las provocadas.

Entre las primeras tenemos: vaciamiento súbito del útero; adelgazamiento de las paredes uterinas por distensión progresiva que pueden llevar al relajamiento de ellas secundario a la atonía total o parcial del órgano (embarazo gemelar, hidramnios); cuello uterino dilatado o relajación del segmento inferior (como sucede en las inserciones bajas de la placenta); inserción fúndica de la placenta; brevedad del cordón; lesiones degenerativas del endometrio; excesiva relajación de ligamentos anchos y redondos; placenta ácreta.

Así, pues, de estas causas las más llamativas son: la atonía del útero, es decir, que el órgano se halle convertido previamente en un saco de paredes flácidas y delgadas y la falta de contracción del orificio interno del cuello con retracción y contracción, simultáneas del cuerpo.

Entre las causas determinantes de la espontánea tenemos: aumento de la presión intra-abdominal por contractura de la pared del abdomen como sucede en el pujo para expulsar la placenta, vómito, tos, estornudo, sentarse estando acostada, dar vueltas en la cama, levantar las caderas para permitir la colocación de un pato o cambio de una sábana, etc.

Las causas determinantes más importantes de las "provocadas" son las "violencias" al efectuar el alumbramiento: método inadecuado de la expresión de Credé; presión suprafúndica aplicada a un útero todavía relajado; extracción de coágulos; tracción sobre el cordón umbilical durante el tercer período del parto y antes del desprendimiento completo de la placenta, con o sin inercia uterina previa; extracción manual de la placenta. De todas éstas la más importante, en el consenso de los autores, son las tracciones del cordón.

El detalle del mecanismo de la inversión es problemático. Algunos creen que él sigue el mismo mecanismo de la intususcepción intestinal.

Lo más común es presumir que hay un área de relajación en el útero, lo más a menudo en el fondo, en el sitio de implantación de la placenta, lo que provoca al retraerse el cuerpo una 
clepresión o hundimiento dentado de dicha porción hacia la cavidad en concomitancia con la contracción activa del músculo uterino restante que la rodea, provocándose entonces la inversión de la manera como se produce la expulsión de un cuerpo extraño, tanto más rápidamente si se provocan cambios en la presión intra-abdominal, se tracciona el cordón o se presiona inadecuaciamente el fondo uterino.

En general se acepta que la inversión uterina aguda es más frecuente en las primíparas: $52 \%$ en la serie de Das. Bell y Wilson (6) en una recopilación de casos de la literatura inglesa y americana de doce años (1941-1953), traen los siguientes datos:

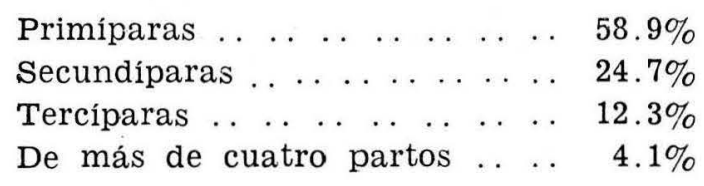

Sin embargo, S. Cosgrave, citado por Curtis (1), al reportar nueve casos encontró que 7 de ellos habían sucedido en multíparas $(77.7 \%)$. Entre nosotros R. Bechara (7) al relatar 7 casos, ya crónicos, operados por él, encontró que ninguno había ocurrido en primíparas.

El caso que nosotros reportamos ocurrió en una primípara.

El accidente puede presentarse en el curso del alumbramiento, en el post-partum inmediato o al segundo, sexto y aun décimoquinto día del puerperio.

En la serie de Das, ya citada, el $72.3 \%$ se presentó durante el tercer período; el $14.2 \%$ durante las primeras 24 horas del puerperio; $9.8 \%$ entre el segundo y trigésimo día del puerperio y cinco casos durante el segundo período. Loizeaux y Mastraioinni (8) han reportado un caso de inversión espontánea a través de la incisión uterina durante una cesárea.

Es muy raro después de abortos o partos prematuros y excepcional, para la mayoría de los autores, como resultado de la tracción de tumores intrauterinos. Conill Montobio (9) sin embargo es muy enfático al afirmar lo contrario, especialmente cuando se trata - dice - de tumores que al crecer adelgazan la musculatura uterina, son peliculados y se insertan en o cerca del fondo pudiendo en tal caso, durante el trabajo automático de expulsión arrastrar el fondo uterino. Es lo que él denomina la inversión aguda ginecológica y denomina a la otra, aguda obstétrica o puerperal. 
Sintomatologia. - Hay un cuarteto sintomático bastante característico, a saber: dolor, shock, hemorragia y masa vaginal.

Una queja de dolor agudo en el hipogastrio (que puede faltar) con sensación de pujo durante el alumbramiento o prontamente en el post-partum, seguido de shock, debe hacer pensar en la posibilidad de inversión uterina aguda. La hemorragia que es otro síntoma cardinal puede faltar en los primeros momentos, pues el shock que es causado por el estiramiento del peritoneo y la compresión de los nervios del ligamento ancho, puede preceder a la hemorragia, especialmente cuando la inversión se produce sin que haya habido desprendimiento de la placenta; e inclusive habiéndose presentado hemorragia el shock es desproporcionado con la pérdida sanguínea.

En algunos casos los síntomas son alarmantes, tanto que pueden acabar rápidamente con la vida de la paciente a pesar clel tratamiento bien establecido; en otros pueden ser ligeros, pasando desapercibidos, tanto que la inversión puede ser reconocída sólo días o semanas después. En este último caso, la inversión permanecerá oculta durante el puerperio, estableciéndose, con completa tolerancia de la paciente, la inversión crónica que a la vuelta de meses y aun de años la conviertan en una ginecópata.

En cuanto a la masa vaginal, puede estar constituída por el útero invertido y la placenta aún adherida, total o parcialmente, si la inversión se produjo durante el tercer período; o sólo el útero cuando se produjo en el post-partum inmediato. Dicha masa puede quedar contenida en la vagina o hacerse visible saliendo fuera de ella. Cuando la placenta se ha desprendido, ya se podrá tactar una masa de superficie irregular de consistencia blanda y un tanto depresible. Si la masa ha salido o se aplica un especulum, se podrá apreciar la superficie endometrial tomentosa, de color rojo vinoso e inclusive se podrán localizar los orificios uterinos de las trompas.

Otros signos de importancia son: la ausencia del cuerpo uterino en su propio sitio y la depresión en forma de embudo que se puede apreciar a la palpación abdominal, después de vaciar la vejiga, y que es de importancia diagnóstica cuando se le reconoce.

Diagnóstico diferencial. - Desde el punto de vista de hemorragia y de shock, debe hacerse con la ruptura uterina, con los desgarros del cuello, con los desgarros vaginales prolongados hacia 
los fondos de saco, con la atonía total del útero. En cuanto a la masa, debe diferenciarse, lo que resulta fácil, del prolapso y de los fibromas uterinos pediculados.

Evolución y pronóstico.- La evolución puede hacerse en tres sentidos: 19 Conduciendo a la muerte por shock y hemorragia; 2 o Corrigiéndose con tratamiento o sin él (algunos casos relatados en la literatura se han desinvertido espontáneamente al cabo de algún tiempo variable), y 3 o Evolucionando hacia la cronicidad relativa o absoluta, siempre y cuando fenómenos infecciosos, necróticos y aún gangrenosos por isquemia debida a retracción exagerada del anillo cervical, no vengan a complicar el cuadro.

El pronóstico no es, ni con mucho, favorable en el conjunto cie los casos reportados, pues la rata de mortalidad (15\% aproximadamente) es muy elevada a pesar de los progresos en el campo transfusional, en el de las drogas anti-shock, de los antibióticos y de los progresos de la técnica obstétrica.

Hay acuerdo general en que el pronóstico depende de el tiempo transcurrido entre la ocurrencia y el diagnóstico, aun no teniendo en cuenta el tipo de tratamiento hecho. Como ejemplo cie este aserto copiamos a continuación el cuadro que traen Bell y Wilson (6), pues habla por sí solo:

Ticmpo de reconocimiento

De 0 a 30 minutos...

De 30 a 60 minutos.

Una a 24 horas... .

24 a 48 horas . . . .

Más de 48 horas... .
Nimero de casos Número de muertes

$\begin{array}{rl}42 & 4 \\ 4 & 1 \\ 17 & 5 \\ 4 & 4 \\ 9 & 0\end{array}$

Porcenta.je

4

$9.5 \%$

$25 \%$

$29.2 \%$

$100 \%$

$0 \%$

Tratamiento.- Se puede dividir en profiláctico y activo.

E! tratamiento profiláctico consiste en: conducción juiciosa Y técnica del tercer período del parto: a) evitando las expresiones (violentas) y las presiones sobre el fondo uterino cuando aún está relajado; b) tratando inmediatamente las atonías uterinas; c) no haciendo tracciones del cordón; d) evitando a la paciente los esfuerzos que provoquen aumento de la presión intra-abdominal. Dexeus Font (10) dice: "La expectativa absoluta de diez minutos con alumbramiento hormonal o farmacológico, es la mejor garantía contra la inversión uterina".

Una buena costumbre en vía del reconocimiento precoz de la inversión uterina aguda, debe ser el T. V. rutinario después 
clel alumbramiento y antes de que la paciente sea llevada a su lecho.

El tratamiento activo comprende: a) el control de la hemorragia por medio de taponamiento de útero dentro de la vagina, con gasa estéril si no se puede proceder a la reducción inmediata; no desprender la placenta cuando aún está adherida sino cuando se esté listo para la reducción manual y con el objeto de producir una disminución del volumen, pues de lo contrario la hemorragia se hará mucho más intensa. Algunos aconsejan reducir la inversión junto con la placenta y efectuar luego el alumbramiento; b) El tratamiento del shock, que debe ser precoz, por los medios de todos conocidos; c) la reposición manual del útero invertido, cuya técnica nos permitimos transcribir, textualmente, del libro de De Lee-Green Hill (4) :

"Se toma en la mano todo el órgano vuelto al revés y con los dedos se abre la porción constreñida del útero, mientras con la palma de la mano se empuja hacia arriba el fondo invertido en la dirección del eje de la pélvis, hacia adelante, para no tropezar con el sacro. Esta maniobra se puede ayudar con la otra mano colocada sobre el abdomen, la cual ayuda a dilatar la porción vuelta al revés. Generalmente lo mejor es dejar la placenta en el útero, si está todavía adherida, pero si es demasiado voluminosa, es necesario desprenderla antes de practicar la reinversión del útero. Conviene reducir el tamaño del cuerpo uterino hinchado, haciendo para ello compresión continua, firme, y uniforme con ambas manos, antes de intentar la reducción. Acaso convenga empujar primero hacia arriba un lado del útero y reponer en primer lugar la porción del órgano que descendió últimamente. Ha de tenerse mucho cuidado de no perforar la pared uterina atónica y blanda. Se puede anestesiar profundamente a la paciente cuando hay propensión al espasmo de la zona constrictiva, y la operación no ha de ser ni prolongada ni forzada";

d) La reposición quirúrgica por vía abdominal.

En frente de la emergencia de una inversión uterina aguda reconocida más o menos precozmente, el plan terapéutico depende cie la forma como la inversión haya repercutido sobre el estado general de la paciente y del estado agudo o subagudo de dicha inversión. De este modo podemos hacer los siguientes grupos: 
19 Shock y hemorragia ligeros o moderados y fase aguda de la inversión (esto es, antes de la retracción del cuello). Reposición manual inmediata, bajo anestesia general y medidas anti-shock. simultáneamente.

20 Shock y hemorragia ligeros o moderados $y$ fase sub-aguda (es decir, después de la retracción del anillo cervical): medidas anti-shock, aplicación de un relajador del anillo de contractura cervical (flaxedil y/o solución de adrenalina al 1 por mil, 1 c. c.) e intento de reposición manual bajo anestesia general profunda. No se debe insistir con bruscos intentos de reducción manual si ésta no es fácil. En tal caso, controlar la hemorragia, continuar con las medidas anti-shock y una vez recuperada la paciente, proceder a la reducción quirúrgica por vía abdominal (técnica de Huntington).

3o Shock intenso, hemorragia abundante y fase aguda o subaguda: medidas anti-shock aún más cuidadosas e intensivas que en los casos anteriores; control de la hemorragia por taponamiento forzado del útero y la vagina. Por ningún motivo practicar, ni siquiera intentar, la reposición manual, pues en estas condiciones la maniobra es con seguridad mortal. Se esperará a que la paciente se recupere del shock, se reemplazará la sangre perdida, y previa la aplicación de relajadores se intentará la reducción manual bajo anestesia general. Si no es fácil, la conducta de escogencia será la reducción quirúrgica por vía abdominal por la técnica de Huntington, cuando se puede llevar a cabo dentro de las primeras 48 horas siguientes a la inversión. Si la recuperación de la paciente a condiciones generales satisfactorias desde el punto de vista quirúrgico sobrepasa las 48 horas, será mejor esperar a que se produzca la involución completa y bajo vigilancia estricta y tratamiento adecuado con el fin de evitar las complicaciones, practicar la reposición quirúrgica por la técnica de Haultain.

Más tarde, y ya dentro de la cronicidad, se le reducirá por la técnica de spinelli o se hará la histerectomía vaginal, según el caso.

\section{CASO REPORTADO}

S. C. A. L.- Carnet número 30817-B. ICSS, Clínica Primero de Mayo.

Primigestante de veintitres años de edad, obesa ( $82 \mathrm{k}$. de peso). Embarazo a término. Ingresa a la clínica el día 12 de octubre de 1954 a las 20:45; había iniciado trabajo 3 horas antes del in- 
greso. Presentación cefálica insinuada; ruidos fetales positivos. T. V.: cuello grueso con 2 a 3 centímetros de dilatación, bolsa integra. Durante la noche del día 12 y el día 13 estuvo con trabajo irregular.

Fue examinada a las 22 horas del dia 13 de octubre de 1954 . encontrándose: presentación cefálica encajada, ruidos fetales buenos, T. V.: cuello borrado, blando, delgado, con 4 a 5 centímetros de dilatación, bolsa íntegra. En vista de que el trabajo ha sido lento e irregular se resuelve estimularlo con Pitocín endovenoso gota a gota ( 5 unidades en 500 c. c. de suero dextrosado al $5 \%$ en agua).

A las 23:30, habiendo recibido 3 unidades de Pitocín, las cuales regularizaron e intensificaron el trabajo: contracciones cada 3 minutos, con 35 a 40 segundos de duración, de buena intensidad y encontrando al T. V. cuello delgado, blando, 8 centímetros de dilatación, bolsa íntegra, OIP. en la exvación, se pasa a la sala de partos. Pulso: 80. T. A.: 124/78. Se rompen artificialmente las membranas y se ordena Trilene durante cada contracción.

A las 0:30 del día 14 se encuentra: presentación descendida hasta el estrecho inferior, dilatación completa, OIA, ruidos fetales un tanto lentos. Se resuelve aplicar forceps para OIA. en el estrecho inferior, previa episiotomía media, bajo anestesia por Trilene. Se obtiene un feto femenino vivo que pesó 3.400 gramos y midió 50 centímetros, en buenas condiciones.

Se espera alrededor de 8 a 10 minutos y en vista de que no se desprende la placenta, se tapona la vagina con una compresa y se procede al cierre de la episiotomía ( 4 planos, sutura intradérmica de la piel; duración, 25 minutos). Al terminar la sutura, 36 minutos después de la expulsión del feto, se saca la compresa y se efectúa el alumbramiento por "extracción simple” (expresión del cuerpo uterino y tracción suave del cordón, con la seguridad de que la placenta estaba ya desprendida). Se aplican 5 unidades de Pitocín disueltas en agua por vía venosa y una ampolla de Ergotrate por vía muscular.

Alrededor de 5 minutos después (tiempo suficiente para despojarnos de las ropas estériles) se pudo apreciar un escurrimiento algo notorio de sangre rutilante por la vagina. Se revisa el globo uterino, pero no se le encuentra. Por ligereza no dimos importancia a esto y creímos que el gran panículo adiposo nos daba una falsa sensación. Pensamos, entonces, en que podría haber un desgarro del cuello, aun cuando no había antecedentes 
para ello, o un desgarro de la vagina, ya que se había aplicado forceps y se trataba de una primípara. Decidimos practicar tacto vaginal para cerciorarnos del estado del cuello y de las paredes vaginales, o de la presencia de un cotiledón retenido. Y cuál sería nuestra sorpresa (porque nunca habíamos tenido oportunidad de ver un caso de éstos ni de haberlo oído relatar), al encontrar la vagina ocupada por una masa de superficie irregular, blanda, ligeramente depresible y del tamaño de una cabeza fetal. Se hace el diagnóstico de inversión uterina aguda; confesamos que no sabíamos la técnica de su tratamiento. La paciente estaba pálida y sudorosa; la tensión arterial había descendido a cifras de $70 / 40$ y el pulso era relativamente lento (82 pulsaciones por minuto).

Como lo lógico era reducir la inversión se volvió a dar anestesia a la paciente y se hizo el intento de la taxis manual, con fracaso; después de esta maniobra la enferma entra en shock completo. Se toman las medidas del caso: transfusión de plasma primero, y luego de sangre total a chorro, calor, posición de Trendelemburg, inyección de 5 c. c. de Escatín y luego Levofed por vía venosa, gota a gota. La hemorragia disminuye un poco, la paciente se recupera moderadamente y con cifras tensionales de $60 / 30$, y nuevamente bajo anestesia por Trilene, el doctor Santiago Lleras intenta nueva maniobra de reducción, fracasando, y la paciente entra nuevamente en shock acentuado.

Se prescinde de nuevas maniobras y se continúa con la aplicación de sangre y Levofed. La hemorragia es ahora escasa.

A las 4 horas de haber descubierto la inversión, las condiciones generales de la paciente son aún poco satisfactorias. Aprovechamos este lapso para ilustrarnos sobre el tratamiento en el texto de De Lee Greenhill.

A las 5 a. m. se encuentra: pulso 100, débil. T. A. 60/40. Se continúa con la transfusión y el Levofed y se resuelve hacer un nuevo intento de reducción manual, con el ánimo predispuesto a que si no era fácil, abordar el problema por la vía abdominal.

Anestesia general (Pentothal, Eter Oxígeno); aplicación de una ampolla de Flaxedil y de 1 c. c. de Solución de Adrenalina al uno por mil. Se lleva a cabo al pie de la letra la maniobra cescrita por De Lee y después de 4 minutos de intentar la maniobra suavemente se logra la reducción de la inversión. Se tapona la cavidad uterina y se aplica una mezcla de 10 unidades de Pitocín y una ampolla de Ergotrate por vía muscular. Se sutura 
nuevamente la episiotomía. Al terminar ésta, la tensión arterial se encuentra en cifras de 10/60. Se deja en observación en la sala ce cirugía y una hora después se le envía a su lecho con T. A. de 110/70. Pulso: 90 y condiciones generales satisfactorias.

Durante el puerperio se le aplicaron ochocientas mil unidades diarias de penicilina y un gramo de estreptomicina, 3 comprimidos diarios de Ergotrate. El taponamiento se retiró 14 horas después. No hubo ninguna complicación y la involución uterina fue absolutamente normal. Cinco días después del parto sale de la clínica en buenas condiciones.

En este caso es de anotar en lo que respecta al diagnóstico que no dimos la importancia debida a la ausencia del globo uterino en su sitio anatómico. $\mathrm{Y}$ en lo que respecta a la conducta que hubo fallas debido a que desconocíamos en detalle la entidad y la manera de tratarla. Se intentó la reducción inmediata sin llenar los requisitos tratándose de shock y hemorragia moderados y fase subaguda; al esperar la recuperación de la paciente no se taponó compresivamente el útero sangrante desde un principio. Aquí tendría cabida lo que anotan Loizeaux y Mastraioinni (8) a este respecto: "Debido a la rareza de la entidad, el tratamiento inmediato es a menudo inadecuado $e$ ineficaz".

Sugerimos que en la producción de esta inversión aguda pudo tener su influencia el efecto relajador que tiene el Trilene sobre el cuerpo uterino, a pesar de la aplicación de Pitocín endovenoso después del alumbramiento, lo cual hacemos de rutina cada vez que damos analgesia o anestesia con Trilene.

No hemos tenido datos sobre otros casos, pero seguramente se han presentado y no se ha hecho el diagnóstico, sobre todo en aquellos casos de shock sorpresivo y fulminante o irreversible y en los que no se halla una causa visible o apreciable. Algunos de esos casos podrían diagnosticarse retrospectivamente: muerte por shock debido a inversión uterina aguda.

Por último, tenemos que anotar que es el primer caso que se presenta entre cerca de 32.000 partos que se han atendido en la Clínica Primero de Mayo del ICSS. 


\section{REFERENCIAS}

1. ARTHUR H. CURTIS.- "Text Book of Gynecology”. Quinta edición. Págs. 657, 661. 1947.

2. TITUS PAUL.- "The Management of Obstetrics Dificulties". Tercera edición. Págs. 571, 574. 1945.

3. PEREZ MANUEL LUIS.- "Compendio de Clínica Obstétrica". Primera edición. Págs. 516, 518. 1949.

4. DE LEE-GREENHILL.- "Principios y Práctica de Obstetricia". Tomo $2^{\circ}$ Págs. 1.028 a 1.036. 1945.

5. G. J. QUIGLEY M. D.- "Inversión o fthe Puerperal Uterus". Am. J. Obst. and Gynec. Vol. 69, número 2. Págs. 277, 283. Febrero de 1955.

6. BELL JAMES E., WILSON FRASER and WILSON LESTER.- "Puerperal Inversion of the Uterus": Am. J. Obst. and Gynec. Vol. 66, número 4. Págs. 767, 777. Octubre de 1953.

7. BECHARA RICARDO.- "Tratamiento Quirúrgico por vía abdominal de la inversión crónica de la matriz". "Revista Colombiana de Obstetricia y Ginecología". Edición especial (Memorias de la Primera Convención $\mathrm{Na}$ cional de Obstetricia y Ginecología). Vol. V, número 5. Págs. 169, 172. Mayo y junio de 1954 .

8. LOIZEAUX LEON S. and LUIGI MASTRAIOINNI.- "Acute Puerperal Inversion of the Uterus". Journal of the American Academy of Obstetrics and Gynecology. Vol. 5, número 2. Págs. 193, 196. Febrero de 1953.

9. CONILL MONTOBIO VICTOR.- "Tratado de Gínecología y de Técnica Terapéutica Ginecológica”. Primera edición. Págs. 521, 524. 1946.

10. DEXEUS FONT S.- "Tratado de Obstetricia". Primera edición. Tomo II. Págs. 1.299, 1.300. 1949. 